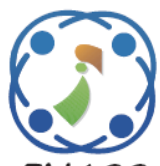

\title{
Multiple Model Adaptive Nonlinear Filter for Range Estimation Using Bearing Only Tracking
}

\author{
Sindhu Babulogiah ${ }^{1 *} \quad$ Valarmathi Jayaraman $^{1}$ \\ ${ }^{I}$ Vellore Institute of Technology, Vellore, India \\ ${ }^{2}$ Denfence Research and Development Organisation, Delhi, India \\ * Corresponding author's Email: bsindhu20@gmail.com
}

Christopher Sargunaraj $^{2}$

\begin{abstract}
This paper discusses about the range estimation using multiple model method for single sensor bearing only tracking (BOT) in 3D. For BOT, the ownship is assumed to take a manoeuvre to gain observability in range and target state. The unknown target range was divided into uniform sub-intervals and nonlinear filters like Extended and unscented Kalman filter (EKF and UKF) with Cartesian and modified spherical coordinates (MSC) were implemented. Comparative results indicate that, UKF in MSC performs better with high computational time. This paper introduces Adaptive nonlinear filter (ANF) to get better range estimate with reduced computational time without affecting the filter performance. It is accomplished by conjoining Cartesian EKF with UKF in MSC, adaptively during the stationary and manoeuvring ownship conditions. The performance comparison was analysed using root mean square error, bias error and computational time. Simulation results reveal that ANF shows better results with reduced computational time.
\end{abstract}

Keywords: Bearing-only tracking, Adaptive nonlinear filter, Modified spherical coordinates, Root mean square error.

\section{Introduction}

The bearing only tracking (BOT) is the most widely used target motion analysis (TMA) in radar, sonar, space surveillance and wireless sensor networks [1-3]. The objective of the paper is to estimate the target range using multiple model method and to estimate the target state. Poor observability and nonlinear measurement model makes BOT a difficult problem [4]. To deal with difficulties, the ownship takes a manoeuvre to gain observability in range and target state [5]. The selection of proper ownship manoeuvring pattern increases the observability, and thereby enhances the tracking performance $[6,7]$. The observability requirements for bearing only tracking are discussed in [8]. For the highly nonlinear measurement model, nonlinear filtering algorithms are used to estimate the target state [9]. The nonlinear filtering algorithms are classified into two types, batch processing and recursive Bayesian approach [10].
This paper focuses on recursive Bayesian filtering approach like EKF and UKF.

Commonly used nonlinear filtering algorithm for BOT is EKF in Cartesian coordinates [11]. But the EKF in Cartesian coordinate causes filter divergence and produces unstable and biased estimates [12]. The problem of filter divergence can be reduced by using EKF in MSC [13, 14]. The advantage of using MSC over Cartesian is that, it automatically allows decomposition into observable and unobservable components of the state estimate and prevents filter instability $[15,16]$. Even though the single model nonlinear filters using MSC show good results, it leads to faulty estimates when initialized poorly and range cannot be inferred [17]. The most appropriate method is to use range parameterized multiple model method (RPMM), in which the unknown range is divided into uniform sub-intervals and nonlinear filters are used in each sub-interval, to identify the best range estimate $[18,19]$. In this paper the nonlinear filters like, EKF in Cartesian (CEKF), EKF in MSC (MSC-EKF), and UKF in Cartesian (CUKF), UKF in MSC (MSC-UKF), were used. 
Among the filters used, MSC-UKF performs better with more computational time. Our aim is to reduce the computational time of MSC-UKF without affecting its performance.

This paper proposes, ANF algorithm to obtain better tracking performance with reduced computational time. The novelty in this paper is, ANF is implemented according to the ownship stationary and manoeuvring conditions. The key idea of the implementation of proposed technique is explained below. The simulation results indicate that during the initial period when ownship is stationary the performance of all nonlinear filters are same and hence CEKF is implemented, since it has low computational time. When the ownship starts a manoeuvre to gain observability it is noticed that, the errors become more and performance of MSCUKF is better since it shows less errors compared to other nonlinear filters. Therefore, MSC-UKF is implemented during the manoeuvring portion of ownship. After the manoeuvre, again the ownship becomes stationary hence CEKF is implemented. Therefore CEKF and MSC-UKF are chosen adaptively based on the ownship stationary and manoeuvring conditions. Simulation results reveal that, ANF performs equally with MSC-UKF with lesser computational time.

The outline of this paper is as follows. Section II describes the dynamic and measurement models of target and ownship in Cartesian coordinate. Section III defines dividing the range uncertainty region and explanations for nonlinear filters and proposed ANF. Section IV discusses the Posterior Cramer Rao lower bound (PCRLB). Section V describes simulation results and comparative analysis of nonlinear filters. Finally, Section VI describes conclusion and future work.

\section{Bearing only tracking}

This section deals with target and ownship geometry for BOT in 3D.

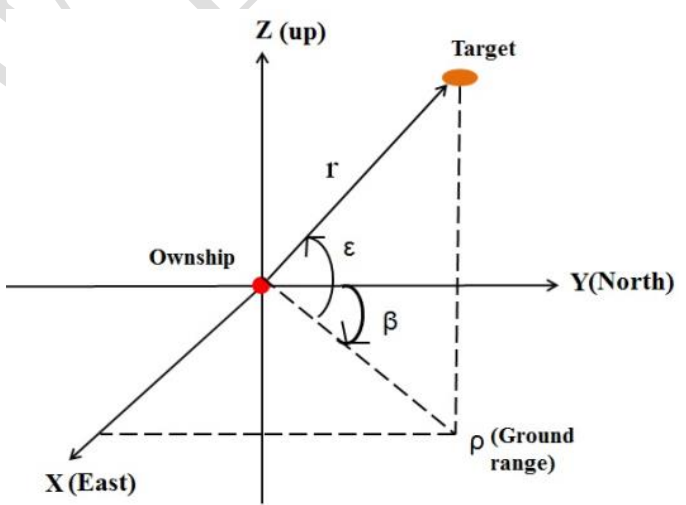

Figure.1 Target and ownship geometry
Fig. 1 represents the target and ownship geometry for BOT. Slant range (r) represents the distance between the target and ownship. The azimuth angle $(\beta)$ is measured clockwise from north and the elevation angle $(\varepsilon)$ is the angle between the ground range $(\rho)$ and slant range $(r)$.

\subsection{Dynamic model and measurement model in Cartesian coordinates}

The dynamic model of the target $\left(x_{k}^{t}\right)$ at time $t_{k}$ is assumed to follow nearly constant velocity (NCV) model and is defined as,

$$
x_{k}^{t}=F_{k, k-1} x_{k-1}^{t}+w_{k, k-1}
$$

where the superscript $t$ defines the dynamic model of the target. $F_{k, k-1}$ represent the transition matrix and $w_{k, k-1}$ is the zero mean, white Gaussian process noise [9].

The transition matrix is defined as [14],

$$
F_{k, k-1}=\left[\begin{array}{cccccc}
1 & 0 & 0 & 1 & 0 & 0 \\
0 & 1 & 0 & 0 & 1 & 0 \\
0 & 0 & 1 & 0 & 0 & 1 \\
0 & 0 & 0 & 1 & 0 & 0 \\
0 & 0 & 0 & 0 & 1 & 0 \\
0 & 0 & 0 & 0 & 0 & 1
\end{array}\right]
$$

The dynamic model of the ownship follows the coordinated turn (CT) model and constant velocity (CV) model. The CT model is used for ownship manoeuvre to observe the target state.

The dynamic model of the ownship $\left(x_{k}^{o}\right)$ at time $t_{k}$ for $\mathrm{CV}$ is described as,

$$
x_{k}^{o}=F_{k, k-1} x_{k-1}^{o}
$$

where $F_{k, k-1}$ is the transition matrix as defined in Eq. (2). The superscript $o$ defines the dynamic model of ownship [12].

The linear dynamic model of the ownship for CT model is defined as,

$$
x_{k}^{o}=F^{C T}(\mathrm{~T}, \omega) x_{k-1}^{o}
$$

where $F^{C T}$ is the transition matrix for CT model and is defined as $[9,17]$, 


$$
F^{C T}=\left[\begin{array}{cccccc}
1 & 0 & 0 & \frac{\sin (\omega, \mathrm{T})}{\omega} & \frac{-[1-\cos (\omega, \mathrm{T})]}{\omega} & 0 \\
0 & 1 & 0 & \frac{[1-\cos (\omega, \mathrm{T})]}{\omega} & \frac{\sin (\omega, \mathrm{T})}{\omega} & 0 \\
0 & 0 & 1 & 0 & 0 & \mathrm{~T} \\
0 & 0 & 0 & \cos (\omega, \mathrm{T}) & -\sin (\omega, \mathrm{T}) & 0 \\
0 & 0 & 0 & \sin (\omega, \mathrm{T}) & \cos (\omega, \mathrm{T}) & 0 \\
0 & 0 & 0 & 0 & 0 & 1
\end{array}\right]
$$

where $\mathrm{T}$ defines the time interval between each measurements and $\omega$ is the turn rate for CT model [16].

The relative state vector in Cartesian coordinates $\left(x_{k}^{c}\right)$ is defined as,

$$
x_{k}^{c}=x_{k}^{t}-x_{k}^{o}
$$

where $x_{k}^{t}$ and $x_{k}^{o}$ are the state vector of the target and ownship [5]. Adding and subtracting of $F_{k, k-1} x_{k-1}^{o}$ on the RHS of Eq. (6) gives,

$$
\begin{gathered}
x_{k}^{c}=F_{k, k-1} x_{k-1}^{t}-F_{k, k-1} x_{k-1}^{o}+w_{k, k-1}-x_{k}^{o}+ \\
F_{k, k-1} x_{k-1}^{o} \\
=F_{k, k-1}\left(x_{k-1}^{t}-x_{k-1}^{o}\right)+w_{k, k-1}-\left(x_{k}^{o}-\right. \\
\left.F_{k, k-1} x_{k-1}^{o}\right) \\
x_{k}^{c}=F_{k, k-1} x_{k-1}^{c}+w_{k, k-1}-U_{k, k-1}
\end{gathered}
$$

where $U_{k, k-1}$ is the deterministic vector which accounts for the effect of mismatch between the ownship and target dynamic model [1,28]. The nonlinear measurement model in Cartesian coordinates $\left(z_{k}\right)$ at time $t_{k}$ is defined as,

$$
\begin{gathered}
z_{k}=h\left(x_{k}^{c}\right)+n_{k} \\
h\left(x_{k}^{c}\right)=\left[\begin{array}{l}
\epsilon_{k} \\
\beta_{k}
\end{array}\right]
\end{gathered}
$$

where $h$ is the nonlinear measurement function. Here, $\epsilon_{k}$ and $\beta_{k}$ are the elevation and azimuth angle measurements and $n_{k}$ is the zero mean white Gaussian measurement noise [4].

\section{Range parameterized multiple model (RPMM) method}

In this paper, RPMM method is implemented to identify the unknown range between the target and ownship. The steps involved in RPMM are given below.
1. The range uncertainty region was divided into $N_{r}$ uniform sub-intervals as shown in Fig. 2, where each sub-interval defines each model.

2. The mean and variance of range are estimated in each sub-interval by assuming that it follows uniform distribution. Using this, the initial state is predicted for the implementation of nonlinear filters in each model.

3. Range is estimated using the predicted range from step 2 along with the measured ranges using nonlinear filters such as EKF-Cart, EKFMSC, UKF-Cart, UKF-MSC and ANF.

4. Initializing equal prior model probability in each sub-interval.

5. The steps 2 and 3 are repeated for the entire observation pertaining to particular range to update the model probability using likelihood function in each sub-interval. As a result of recursion, the maximum probability occurs in the model which has true range of the target and low probability for other models.

6. The final step is the fusion of estimates from all the models to obtain the optimized estimate.

\subsection{Division of range uncertainty region}

The superior performance of the RPMM can be achieved by dividing the large range uncertainty region into uniform sub-intervals [19]. Since the range estimate is not known accurately during the initialization, considering the unknown range region lies between $\left(r_{\min }, r_{\max }\right)$ and $N_{r}$ filters are used for tracking.

Fig. 2 describes dividing the range uncertainty region into uniform sub-intervals as stated in step 1 . The midpoint of each sub-interval is taken as the mean and is described as [20],

$\bar{r}_{j}=\frac{\Delta_{r}}{2}+(j-1) \Delta_{r} \quad j=1,2, \ldots \ldots N_{r}$

where $\bar{r}_{j}$ defines the mean of the $j^{\text {th }}$ sub-interval.

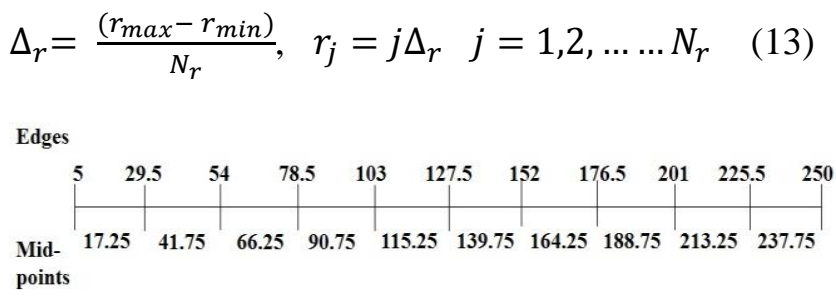

Figure.2 Range interval division 
Where $r_{j}$ defines the edges of each sub-interval as shown in Fig. 2. The variance of each sub-interval is defined as,

$$
\sigma_{r}^{2}=\frac{\Delta_{r}^{2}}{12}
$$

In each sub-interval the nonlinear filters are implemented to identify which sub-interval gives the best estimate of the range as stated in step 2 and 3.

\subsection{Initial state and covariance estimate of nonlinear filters in Cartesian and MSC}

The initial state estimate $\left(\hat{x}_{k-1 \mid k-1, j}^{c}\right)$ and covariance $\left(P_{k-1 \mid k-1, j}^{c}\right)$ at time $t_{k-1}$ for $j^{\text {th }}$ subinterval in Cartesian coordinates is given as,

$$
\hat{x}_{k-1 \mid k-1, j}^{c}=\left[\begin{array}{c}
x \\
y \\
z \\
\dot{x} \\
\dot{y} \\
\dot{z}
\end{array}\right]=\left[\begin{array}{c}
\bar{r}_{j} \cos (\epsilon) \sin (\beta) \\
\bar{r}_{j} \cos (\epsilon) \cos (\beta) \\
\bar{r}_{j} \sin (\epsilon) \\
s \cos (\dot{\epsilon}) \sin (\dot{\beta})-\dot{x}^{o} \\
s \cos (\dot{\epsilon}) \cos (\dot{\beta})-\dot{y}^{o} \\
\sin (\dot{\epsilon})-\dot{z}^{o}
\end{array}\right]
$$

Where $\bar{r}_{j}$ defines the mean range for each subinterval as given in Eq. (12). $\epsilon$ and $\beta$ are the elevation and azimuth angle measurements and $\dot{\epsilon}$ and $\dot{\beta}$ are the elevation and azimuth velocity component [9].

$$
\begin{aligned}
& P_{k-1 \mid k-1, j}^{c}= \\
& {\left[\begin{array}{rllllll}
P_{x x} & P_{x y} & P_{x z} & 0 & 0 & 0 \\
P_{y x} & P_{y y} & P_{y z} & 0 & 0 & 0 \\
P_{z x} & P_{z y} & P_{z z} & 0 & 0 & 0 \\
0 & 0 & 0 & P_{\dot{x} \dot{x}} & P_{\dot{x} \dot{y}} & P_{\dot{x} \dot{z}} \\
0 & 0 & 0 & P_{\dot{y} \dot{x}} & P_{\dot{y} \dot{y}} & P_{\dot{y} \dot{z}} \\
0 & 0 & 0 & P_{\dot{z} \dot{x}} & P_{\dot{z} \dot{y}} & P_{\dot{z} \dot{z}}
\end{array}\right]}
\end{aligned}
$$

The elements of the covariance matrix with detailed derivation are defined in $[4,16]$.

Similarly for MSC, the initial state estimate $\left(\hat{x}_{k-1 \mid k-1, j}^{m s c}\right)$ and covariance $\left(P_{k-1 \mid k-1, j}^{m s c}\right)$ at time $t_{k-1}$ for $j^{t h}$ sub-interval are defined as $[13,14]$,

$$
\hat{x}_{k-1 \mid k-1, j}^{m s c}=\left[\begin{array}{llllll}
\epsilon & \dot{\epsilon} & \beta & \dot{\beta} & \dot{\xi} & \frac{1}{\bar{r}_{j}}
\end{array}\right]^{\prime}
$$

Here $\dot{\xi}=\frac{\dot{r}}{r}$ is the range rate divided by range and the rest of the components are defined in Eq. (15).

$$
P_{k-1 \mid k-1, j}^{m s c}=\operatorname{diag}\left[\begin{array}{llllll}
\sigma_{\epsilon}^{2} & \sigma_{\dot{\varepsilon}}^{2} & \sigma_{\beta}^{2} & \sigma_{\dot{\beta}}^{2} & \sigma_{\dot{\xi}}^{2} & \sigma_{\frac{1}{\bar{r}_{j}}}^{2}
\end{array}\right]^{\prime}
$$

Similarly, Eq. (18) defines the variances for the components defined in Eq. (17).

\subsection{Nonlinear filters in Cartesian coordinates}

EKF and UKF are the most widely used nonlinear filters for BOT. Both apply the standard Kalman filter methodology, considering the nonlinear measurements [24].

\subsubsection{EKF}

EKF uses the Taylor's series approximation to linearize the nonlinear measurement model [29]. The predicted state estimate $\left(\hat{x}_{k \mid k-1, j}^{c}\right)$ and covariance $\left(P_{k \mid k-1, j}^{c}\right)$ is given by,

$$
\begin{aligned}
& \hat{x}_{k \mid k-1, j}^{c}=F_{k, k-1} \hat{x}_{k-1 \mid k-1, j}^{c}-U_{k, k-1} \\
& P_{k \mid k-1, j}^{c}=F_{k, k-1} P_{k-1 \mid k-1, j}^{c} F_{k, k-1}^{\prime}+Q_{k, k-1}
\end{aligned}
$$

Where $j=1,2, \ldots \ldots, N_{r}$ defines the number of subintervals. $\hat{x}_{k-1 \mid k-1, j}^{c}$ and $P_{k-1 \mid k-1, j}^{c}$ are the initial state estimate and covariance as defined in Eq. (15) and Eq. (16).

The nonlinear predicted measurement $\left(\hat{z}_{k \mid k-1, j}\right)$ is given by,

$$
\begin{aligned}
& \hat{z}_{k \mid k-1, j} \approx h_{k}\left(\hat{x}_{k \mid k-1, j}^{c}\right) \\
& H_{k}=\frac{\partial h_{k}\left(x_{k}\right)}{\partial x_{k}} \mid x_{k}=\hat{x}_{k \mid k-1, j}^{c}
\end{aligned}
$$

Where $H_{k}$ is the jacobian of the nonlinear measurement function $h_{k}$. [24],

The innovation and its covariance are given by

$$
\begin{aligned}
& \vartheta_{k, j}=z_{k, j}-\hat{z}_{k \mid k-1, j} \\
& S_{k, j}=H_{k} P_{k \mid k-1, j}^{c} H_{k}^{\prime}+R_{k}
\end{aligned}
$$

The gain matrix is given by,

$$
K_{k, j}=P_{k \mid k-1, j}^{c} H_{k}^{\prime}\left[H_{k} P_{k \mid k-1, j}^{c} H_{k}^{\prime}+R_{k}\right]
$$

The updated state estimate $\left(\hat{x}_{k \mid k, j}^{c}\right)$ and its covariance $\left(P_{k \mid k, j}^{c}\right)$ are given by,

$$
\begin{aligned}
\hat{x}_{k \mid k, j}^{c} & =\hat{x}_{k \mid k-1, j}^{c}+K_{k, j}\left(z_{k}-\hat{z}_{k \mid k-1, j}\right) \\
P_{k \mid k, j}^{c} & =\left[I-K_{k, j} H_{k}\right] P_{k \mid k-1, j}^{c}
\end{aligned}
$$




\subsubsection{UKF}

The high degree of nonlinearity degrades the performance of EKF and the derivation of Jacobian matrices leads to difficulties in implementation, in such cases UKF is used. It uses the deterministic sigma point calculation to linearize the nonlinear measurement model [22, 27].

The initial state estimate and covariance is same as Eq. (15) and Eq. (16). The predicted state estimate $\left(\hat{x}_{k \mid k-1, j}^{c}\right)$ is given by,

$$
\hat{x}_{k \mid k-1, j}^{c}=\sum_{i=0}^{2 n} W_{m}^{i} x_{k \mid k-1, j}^{c, i}
$$

where $W_{m}^{i}$ is the weighted sample mean and is defined in Eq. (30) and Eq. (31) and $x_{k \mid k-1, j}^{c, i}$ is the predicted sigma points [29].

\subsubsection{Sigma point calculation}

For $\mathrm{n}$ dimensional state vector, $2 \mathrm{n}+1$ sigma points are generated. The BOT considered in this paper, has $\mathrm{n}=6$, hence 13 sigma points are generated based on the following conditions [17],

$$
\begin{aligned}
& x_{k-1 \mid k-1, j}^{c, i} \hat{x}_{k-1 \mid k-1, j}^{c} \\
& \begin{cases}\hat{x}_{k-1 \mid k-1, j}^{c}+\left(\sqrt{(n+\lambda) P_{k-1 \mid k-1, j}^{c}}\right)_{i}, & i=1,2, \ldots n \\
\hat{x}_{k-1 \mid k-1, j}^{c}-\left(\sqrt{(n+\lambda) P_{k-1 \mid k-1, j}^{c}}\right)_{i}, & i=1,2, \ldots .2 n\end{cases}
\end{aligned}
$$

where $\mathrm{i}$ refers to the number of sigma points and $\mathrm{j}$ refers to the number of sub-intervals.

\subsubsection{Weight vector calculation}

The weight vector for mean $\left(W_{m}^{i}\right)$ and covariance $\left(W_{c}^{i}\right)$ is given by $[9,22]$,

$$
\begin{aligned}
& W_{m}^{0}=\frac{\lambda}{(n+\lambda)} \quad i=0, \\
& W_{m}^{i}=W_{c}^{i}=\frac{1}{2(n+\lambda)} \quad i=1,2, \ldots \ldots, 2 n
\end{aligned}
$$

where $\lambda$ is the scaling parameter and is defined as,

$$
\lambda=\alpha^{2}(n+\kappa)-n
$$

where $\alpha$ and $\kappa$ are constants. The value of $\alpha$ is chosen between $1 e^{-4} \leq \alpha \leq 1$ and $\kappa$ is set to zero [27]. Once the predicted state is calculated based on Eq. (28), the rest of filter equations follow the same procedure as Cartesian EKF given in Eq. (23) to Eq. (27) based on the nonlinear predicted measurement defined in Eq. (21).

\subsection{Nonlinear filters in MSC and LSC}

For the nonlinear filter EKF using Cartesian coordinates, the target position and velocity is unobservable, if there is no change in the relative velocity [13, 17]. The linearization of nonlinear measurement model for CEKF depends on the partial derivative of the Jacobian matrix with respect to the state vector defined in Eq. (22). This process leads to biased estimates and filter divergence due to poor observability $[4,5]$. To reduce this difficulty, the modified spherical coordinates (MSC) was proposed by [13]. This coordinate is widely used and much suitable for AOT, because first four components of state vector defined in Eq. (17) directly involves the azimuth and elevation angle measurements and its derivatives and is always observable [28,29]. The range information is obtained upon ownship manoeuvre, the unobservability in range before the ownship manoeuvre does not degrade the performance, because range information can be obtained from first four components [25]. Thus, nonlinear filters using MSC reduces the filter divergence and produces stable and unbiased estimates [5].

\subsubsection{State vector and measurement process in MSC} by,

The relative state vector in MSC $\left(x_{k}^{m s c}\right)$ is given

$$
x_{k}^{m s c}=\left[\begin{array}{llllll}
\epsilon & \dot{\epsilon} & \beta & \omega & \dot{\xi} & \frac{1}{r}
\end{array}\right]^{\prime}
$$

where $\omega=\dot{\beta} \cos (\varepsilon), \dot{\xi}=\frac{\dot{r}}{r}, \epsilon$ and $\beta$ are the elevation and azimuth angle measurements.

The measurement model in MSC is linear, since bearing and azimuth are components of MSC and is defined as $[3,4]$,

$$
z_{k}=H_{k} x_{k}^{m s c}+n_{k}
$$

where $H_{k}$ is the linear measurement matrix and $n_{k}$ is the zero mean white Gaussian measurement noise.

\subsubsection{EKF in MSC}

The filtering using MSC, involves the nonlinear dynamic model and linear measurement model [3]. The linearization of nonlinear dynamic model makes the process difficult, since it requires complex differential manipulations and it is not easy to obtain 
equal representation as in Cartesian coordinates [15]. Due to this difficulty, the alternate method is to use the conversion matrix in the predicted state estimate $[4,28]$. The details regarding the conversion matrix and its equations are given in [21]. The initial state estimate and covariance at time $t_{k-1}$ is defined in Eq. (17) and Eq. (18). The initial state is converted to Cartesian and it is predicted to time $t_{k}$ and again it is converted back to MSC using a conversion matrix as stated in Eq. (35) to use it in a nonlinear filter. The equation for predicted state is given below.

The predicted state estimate $\left(\hat{x}_{k \mid k-1, j}^{m s c}\right)$ at time $t_{k}$ is defined as [16],

$$
\hat{x}_{k \mid k-1, j}^{m s c}=f_{c}^{m s c}\left[F_{k, k-1} \hat{x}_{k-1 \mid k-1, j}^{c}-U_{k, k-1}\right]
$$

The rest of the filter equations are defined in [21].

\subsubsection{UKF in MSC}

The initial state estimate and covariance of UKF-MSC follows Eq. (17) and Eq. (18). The UKF in MSC follows the same steps as UKF-Cart, except it uses Eq. (17) for sigma point calculation. As stated earlier for EKF-MSC in Eq. (35), the UKFMSC also uses the conversion matrix in the predicted state estimate with the weight vector defined in Eq. (30) and Eq. (31). The rest, follows the same procedure as UKF in Cartesian.

\subsection{Adaptive nonlinear filter (ANF)}

The proposed algorithm was introduced to reduce the computational time of the filtering process. As discussed earlier in section 1, the comparative results indicate that MSC-UKF has less error compared to other filters at the time of ownship manoeuvre. It is also noticed that, MSCUKF performs better with high computational time, because of sigma point calculation and conversion matrix in the predicted state estimate. Our aim is to achieve the better performance with low computational time. Hence, ANF is proposed to achieve the better performance with low computational time. The steps involved in ANF are given below.

ANF is implemented, based on the stationary and manoeuvring conditions of the ownship as shown in Fig. 3.

In each sub-interval, during the initial period when the ownship is stationary CEKF is implemented using the filter equations described in subsection 3.3.1.
When the ownship starts a manoeuvre as shown in Fig. 3, MSC-UKF is implemented as described in subsection 3.4 .3 by considering the current updated state estimate and covariance of CEKF as its initial state estimate and covariance.

After gaining the observability of target state, again the ownship becomes stationary during this time period CEKF is implemented as described in subsection 3.3.1 with current updated state estimate and covariance of UKF-MSC as the initial state estimate and covariance for CEKF.

The steps 3 and 4 are repeated recursively in each sub-interval based on the ownship stationary and manoeuvring conditions.

Thus the simulation results indicate that, adaptive combination of CEKF and MSC-UKF effectively reduces the computational time and achieves the performance similar to MSC-UKF.

\subsection{Model probability calculation}

As stated earlier in step 4 of section 3, the prior model probability $P\left(j \mid Z_{k-1}\right)$ in each sub-interval is defined as,

$$
P\left(j \mid Z_{k-1}\right)=\frac{1}{N_{r}}
$$

where $P\left(j \mid Z_{k-1}\right)$ is the probability of model $\mathrm{j}$ being correct among the models considered, given the measurements up to time $t_{k-1}$ [20].

As stated in step 5 of section 3, the model probability at time $t_{k}$ is computed recursively using Baye's rule and is defined as [1],

$$
\begin{array}{r}
P\left(j \mid Z_{k}\right) \propto P\left(z_{k} \mid j, Z_{k-1}\right) P\left(j \mid Z_{k-1}\right) \\
j=1,2, \ldots N_{r}
\end{array}
$$

where $P\left(j \mid Z_{k}\right)=w_{k, j}$ is the model probability or weight of the $j^{\text {th }}$ filter. $P\left(j \mid Z_{k-1}\right)$ in the R.H.S of Eq. (37) is the prior model probability as described in Eq. (36) and $P\left(z_{k} \mid j, Z_{k-1}\right)$ defines the likelihood of model $\mathrm{j}$ at time $t_{k}$ given the measurements up to time $t_{k-1}$. Each time the likelihood is used to update the model probability and is defined as [2,17],

$$
\begin{aligned}
P\left(z_{k} \mid j, Z_{k-1}\right)= & \aleph\left(z_{k}-\hat{z}_{k \mid k-1} ; 0_{2 \times 1}, S_{k, j}\right) \\
& =\aleph\left(\vartheta_{k, j} ; 0_{2 \times 1}, S_{k, j}\right)
\end{aligned}
$$

here, $\vartheta_{k, j}$ and $S_{k, j}$ are the innovation and its covariance of the nonlinear filter from Eq. (23) and Eq. (24) matched to model j.

Each sub-interval has the state estimate and covariance obtained by the $j^{\text {th }}$ filter. As stated in step 6 of section 3, the best estimate is obtained by 
combining the model probability with the state estimate and covariance of all the sub-intervals. The combined state estimate and covariance are given by [9],

$$
\begin{gathered}
\hat{x}_{k \mid k}=\sum_{j=1}^{N_{r}} w_{k, j} \hat{x}_{k \mid k, j} \\
P_{k \mid k}=\sum_{j=1}^{N_{r}} w_{k, j}\left[P_{k \mid k, j}+\left(\hat{x}_{k \mid k, j}-\hat{x}_{k \mid k}\right)\left(\hat{x}_{k \mid k, j}-\right.\right. \\
\left.\left.\hat{x}_{k \mid k}\right)^{\prime}\right]
\end{gathered}
$$

\section{Posterior Cramer Rao lower bound (PCRLB)}

The posterior Cramer Rao lower bound (PCRLB) refers to the bound on the best achievable accuracy and useful method of checking the performance of an unbiased estimator [30]. The unbiased estimator $\hat{x}_{k \mid k}$ from Eq. (40) of the target state $x_{k}$, with the sequence of measurements $Z_{k}=$ $\left\{z_{1}, z_{2}, \ldots \ldots, z_{n}\right\}$. The unbiased estimator has the covariance matrix $P_{k \mid k}$ represented in Eq. (41) and it has the lower bound represented as,

$$
P_{k \mid k}=E\left[\left(\hat{x}_{k \mid k}-x_{k}\right)\left(\hat{x}_{k \mid k}-x_{k}\right)^{\prime}\right] \geq J_{k}^{-1}
$$

where $J_{k}$ refers to the fisher information matrix (FIM) and its inverse is referred to as PCRLB. The difference between $P_{k \mid k}-J_{k}^{-1}$ is the positive semi definite matrix. The recursive formula for FIM was given by [44, 23].

For the nonlinear filtering problem, considering linear dynamic model, additive Gaussian noise and measurement process the recursive formula reduces to [22],

$$
\begin{gathered}
J_{k+1}=Q_{k}^{-1}+H_{k+1}^{T} R_{k+1}^{-1} H_{k+1}-Q_{k}^{-1} F_{k}\left(J_{k}+\right. \\
\left.F_{k}^{T} Q_{k}^{-1} F_{k}\right)^{-1} F_{k}^{T} Q_{k}^{-1}
\end{gathered}
$$

Using matrix inversion lemma Eq. (43) can be written as,

$J_{k+1}=\left(Q_{k}+F_{k} J_{k}^{-1} F_{k}^{T}\right)^{-1}+\left[H_{k+1}^{T} R_{k+1}^{-1} H_{k+1}\right]$

The detailed derivations of the FIM are given in [17, 30].

\section{Results and discussion}

In this section, the performance analysis of all the nonlinear filters using range parameterized multiple model (RPMM) method are compared. The target and ownship geometry are given in Fig. 3. The target is assumed to follow nearly constant velocity

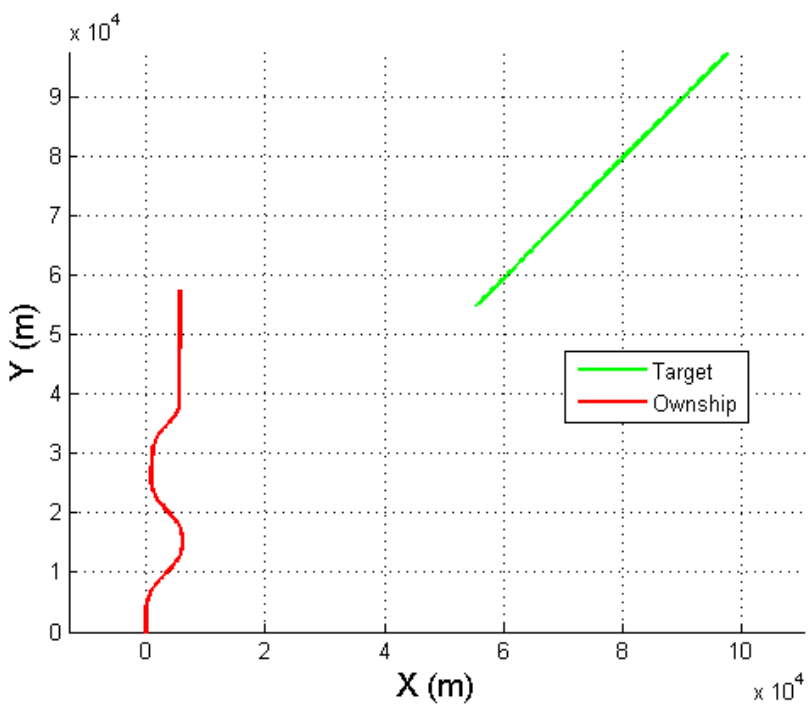

Figure.3 Target and ownship geometry

(NCV) model. Since the ownship takes a manoeuvre to observe the state of the target, it follows constant velocity (CV) and coordinated turn (CT) models [16]. The ownship is assumed to move at the height of $10 \mathrm{~km}$ and the initial target height is assumed to be $9 \mathrm{~km}$. The measurement error standard deviations were assumed to be 0.015 radian for bearing and elevation measurements with the measurement sampling interval of $1.0 \mathrm{~s}$.

The initial true range of the target is assumed to be $138 \mathrm{~km}$ at the line of sight (LOS) of $45 \mathrm{deg}$ and the elevation angle was assumed to be $5 \mathrm{deg}$. The speed of the target and ownship is assumed to be $297(\mathrm{~m} / \mathrm{s})$. Initially for RPMM, the unknown range region $\left[r_{\text {min }}, r_{\text {max }}\right]$ was chosen between $[5 \mathrm{~km}$, $250 \mathrm{~km}]$ and it is divided into $N_{r}=10$ uniform subintervals. In each sub-interval 0.1 equal weights (model probability) was assumed as stated in Eq. (36). The simulations were repeated for 100 Monte Carlo runs.

Table. 1 represents the average values of model probabilities defined in Eq. (37), using different nonlinear filters for the mean range defined in each sub-interval as shown in Fig.2. For all filters the highest probability corresponds to $139.75 \mathrm{~km}$, which is much closer to the true range of $138 \mathrm{~km}$. For other range values the probability is very low, this indicates that all the filters, including ANF show the correct range estimate.

Simulations were also analysed for increased number of sub-intervals, but it is noticed that, even with increased sub-intervals the accuracy of results remains same with increased computational time. Hence we have restricted ourselves with 10 subintervals. The performance of the RPMM is 
Table 1. Model probabilities for different nonlinear filters

\begin{tabular}{|c|c|c|c|c|c|}
\hline Range & CEKF & MSC-EKF & CUKF & MSC-UKF & ANF \\
\hline $17.25 \mathrm{~km}$ & 0.0500 & 0.0051 & 0.0479 & 0.0001 & 0.0370 \\
\hline $41.75 \mathrm{~km}$ & 0.2372 & 0.0074 & 0.0578 & 0.0041 & 0.0082 \\
\hline $66.25 \mathrm{~km}$ & 0.3093 & 0.0272 & 0.0754 & 0.0211 & 0.0052 \\
\hline $90.75 \mathrm{~km}$ & 0.2282 & 0.0523 & 0.0846 & 0.1387 & 0.0197 \\
\hline $115.25 \mathrm{~km}$ & 0.1140 & 0.0792 & 0.0925 & 0.1477 & 0.1142 \\
\hline $139.75 \mathrm{~km}$ & 0.9843 & 0.9937 & 0.9865 & 0.9927 & 0.9916 \\
\hline $164.25 \mathrm{~km}$ & 0.0134 & 0.1391 & 0.1146 & 0.0665 & 0.1043 \\
\hline $188.75 \mathrm{~km}$ & 0.0037 & 0.1694 & 0.1278 & 0.0538 & 0.0627 \\
\hline $213.25 \mathrm{~km}$ & 0.0177 & 0.1961 & 0.1415 & 0.0463 & 0.0646 \\
\hline $237.75 \mathrm{~km}$ & 0.0096 & 0.2160 & 0.1553 & 0.0262 & 0.0484 \\
\hline
\end{tabular}

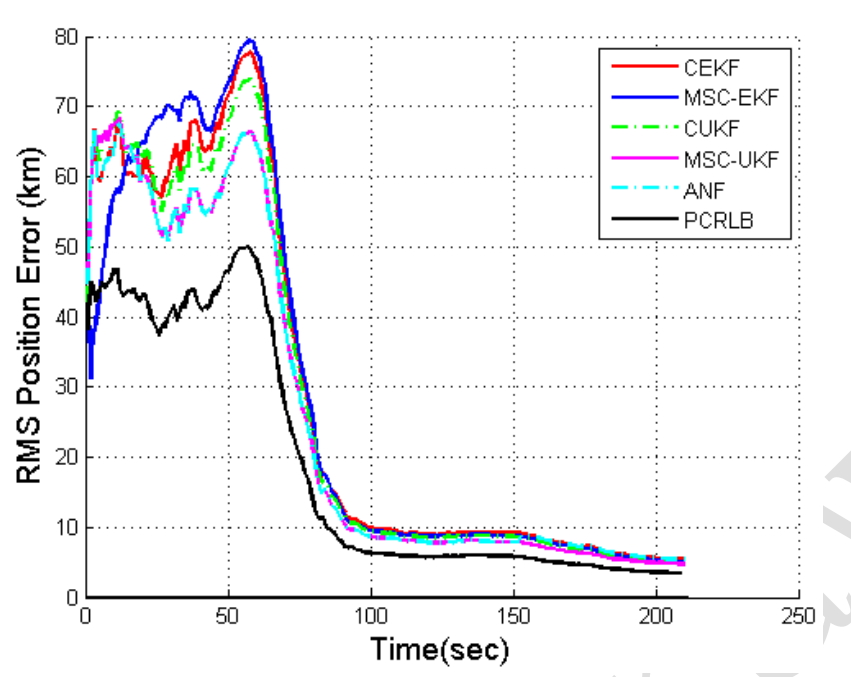

Figure.4 RMS position error

evaluated using RMSE compared with the PCRLB, bias error and computational time.

The RMSE is the measure of filter performance and is defined as [29],

$$
R M S E=\sqrt{\frac{1}{M} \sum_{m=1}^{M}\left\|x_{k, m}-\hat{x}_{k \mid k, m}\right\|^{2}}
$$

where $x_{k, m}$ is the true state and $\hat{x}_{k \mid k, m}$ is the updated state from Eq. (40) in the $m^{\text {th }}$ Monte Carlo run and $\mathrm{M}$ is the number of Monte Carlo runs.

Fig. 4 and 5 denotes the RMS position and velocity errors for the RPMM using different nonlinear filters compared with the PCRLB. It is noticed that, large errors during the initial period of tracking are due the deficiency of a priori knowledge of the initial target range. As the measurements are increased, the error becomes less and filters gradually attain PCRLB.

As stated earlier in subsection 3.5 it is observed

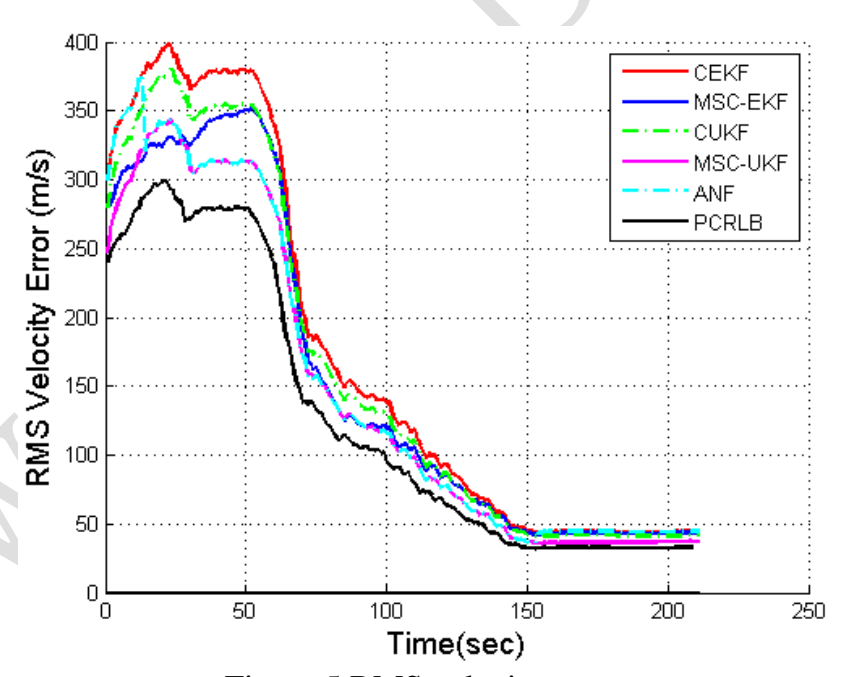

Figure.5 RMS velocity error

Table 2. RMS position and velocity error

\begin{tabular}{|l|l|l|}
\hline Filter & $\begin{array}{l}\text { Position } \\
\text { RMSE(km) }\end{array}$ & $\begin{array}{l}\text { Velocity } \\
(\mathbf{m} / \mathbf{s})\end{array}$ \\
\hline CEKF & 9.5968 & 55.6369 \\
\hline MSC-EKF & 7.6863 & 53.2776 \\
\hline CUKF & 8.1647 & 55.1431 \\
\hline MSC-UKF & 5.3817 & 35.3292 \\
\hline ANF & $\mathbf{5 . 7 7 5 7}$ & $\mathbf{3 9 . 9 7 0 0}$ \\
\hline
\end{tabular}

from Fig. 4 and 5 that, ANF follows MSC-UKF during the ownship manoeuvre and CEKF during ownship stationary. The comparison between the nonlinear filters indicates that, MSC-UKF and ANF shows less RMS errors compared to other nonlinear filters during the ownship manoeuvre and the errors are nearly same for all the nonlinear filters during the ownship stationary.

Table. 2 indicates the numerical values for RMS position and velocity errors for all the nonlinear filters. The RMS error values are less for MSC-UKF and ANF, compared to other filters. The error values 
Table 3. Position and velocity bias error

\begin{tabular}{|c|c|c|c|}
\hline Filter & Position bias (km) & & $\begin{array}{l}\text { Velocity bias } \\
(\mathbf{m} / \mathbf{s})\end{array}$ \\
\hline CEKF & $\begin{array}{ll}{[3.4925} & 1.2626 \\
0.0841] & \end{array}$ & - & $\begin{array}{ll}{[14.3935} & 25.2601 \\
-0.1082] & \end{array}$ \\
\hline $\begin{array}{l}\text { MSC- } \\
\text { EKF }\end{array}$ & $\begin{array}{ll}{[2.8516} & 2.0788 \\
0.0506] & \\
\end{array}$ & - & $\begin{array}{ll}13.2217 & 13.8392 \\
-0.6407] & \\
\end{array}$ \\
\hline CUKF & $\begin{array}{ll}2.4181 & 1.6511 \\
0.0470] & \\
\end{array}$ & - & $\begin{array}{ll}{[11.3681} & 6.5588 \\
-0.5320] & \\
\end{array}$ \\
\hline $\begin{array}{l}\text { MSC- } \\
\text { UKF }\end{array}$ & $\begin{array}{ll}{[1.5712} & 1.3337 \\
0.0318] & \end{array}$ & - & $\begin{array}{l}{\left[\begin{array}{lll}3.1338 & 11.9442 & - \\
0.4806\end{array}\right]}\end{array}$ \\
\hline ANF & $\begin{array}{ll}{[1.6460} & 1.3672 \\
0.0370] & \\
\end{array}$ & & $\begin{array}{ll}5.9141 & 15.6686 \\
-0.4914] & \\
\end{array}$ \\
\hline
\end{tabular}

Table 4. Computational time

\begin{tabular}{|l|l|}
\hline \multicolumn{1}{|c|}{ Filters } & \multicolumn{1}{c|}{$\begin{array}{c}\text { Computational } \\
\text { time(sec) }\end{array}$} \\
\hline CEKF & 0.7750 \\
\hline MSC-EKF & 1.3556 \\
\hline CUKF & 1.4113 \\
\hline MSC-UKF & 8.6920 \\
\hline ANF & $\mathbf{7 . 0 7 7 2}$ \\
\hline
\end{tabular}

for MSC-UKF and ANF are nearly same which indicates that, performance of ANF is similar to MSC-UKF.

The second parameter used to analyse the performance of nonlinear filters is bias error and is defined as [9],

$$
\bar{e}_{k}=\frac{1}{M} \sum_{m=1}^{M} e_{k, m}
$$

Where $e_{k, m}=x_{k, m}-\hat{x}_{k \mid k, m}$, is the error as defined in Eq. (45).

Table. 3 represent the numerical results for position and velocity bias errors along $\mathrm{X}, \mathrm{Y}$ and $\mathrm{Z}$ axis for all the nonlinear filters. Since there is no movement of target along $\mathrm{Z}$ axis, the bias error is very small. Among the filters used, the position and velocity bias errors for MSC-UKF and ANF are comparatively low than other nonlinear filters. The comparison between MSC-UKF and ANF indicates that, the error values are nearly same and ANF performs similar to MSC-UKF.

The computational time for all the filters are given in Table 4. It is calculated using tic, toc function in matlab programming which was executed on Pentium(R) Dual-core CPU T4300 at 2.10GHZ with 3GB RAM. From Table 4. MSCUKF has high computational time than other filters. This is due to the sigma point conversion from MSC to Cartesian and vice versa in the predicted state. It is noticed that, the computational time of the proposed technique ANF is less compared to MSCUKF. This is due to the adaptive combination of
CEKF and MSC-UKF. This reveals that ANF is efficient in terms of computational time and effective in terms of achieving better performance similar to MSC-UKF. The computational time for CEKF, MSC-EKF and CUKF are low, but performances are not better than MSC-UKF and ANF.

\section{Conclusion and future work}

In this paper, ANF is introduced to achieve better performance with lesser computational time. The simulation results indicate that multiple model approach is effective, because it divides the large range uncertainty region into uniform sub-intervals. The simulation results reveal that, MSC-UKF and ANF performs better than other nonlinear filters used. The comparison between the MSC-UKF and ANF illustrates that, ANF performs similar to MSCUKF interms of RMS error and bias error and efficient interms of computational time. In future, ANF can also be applied for target manoeuvring scenarios. Since ANF involves two nonlinear filters, the switching between the filters and the initial estimates given to one filter to the other may also lead to incorrect estimates this should be taken care in future.

\section{References}

[1] S. Arulampalam and B. Ristic, "Comparison of the Particle Filter with Range-Parameterised and Modified Polar EKFs for Angle-Only Tracking", In: Proc. of Signal and Data Processing of Small Targets, Orlando, pp.288-299, 2000.

[2] R. Karlsson and F. Gustafsson, "Range estimation using angle-only target tracking with particle filters", In: Proc. of American Control Conference, Arlington, pp. 3743-3748, 2001.

[3] S.D. Gupta, J.Y. Yu, M. Mallick, M. Coates, and M. Morelande, "Comparison of Angle-only Filtering Algorithms in 3D Using EKF, UKF, PF, PFF, and Ensemble KF", In: Proc. of 18th International Conference on Information Fusion, Washington,USA, pp. 1649-1656, 2015.

[4] M. Mallick, V. Krishnamurthy, and B. Ngu Vo, Integrated tracking classification and sensor management: Theory and Applications, Wiley, IEEE press, 2012.

[5] B.L. Scala and M. Morelande, "An analysis of the single sensor bearings-only tracking problem", In: Proc. of the 11th International Conf. on Information Fusion, Cologne, Germany, pp.1-6, 2008.

[6] J.P. Le Cadre, "Optimization of the Observer Motion for Bearings-Only Target Motion 
Analysis", In: Proc. of 36th Conf. on Decision and Control, San Diego, California, pp. 31263131, 1997.

[7] M.T. Sabet, A.R. Fathi, and H.R.M. Daniali, "Optimal design of the Own Ship maneuver in the bearing-only target motion analysis problem using a heuristically supervised Extended Kalman Filter", Ocean Engineering, Vol. 123, pp. 146-153, 2016.

[8] S.C. Nardone and V.J. Aidala, "Observability Criteria For Bearings-Only Target Motion Analysis", IEEE Transactions On Aerospace And Electronic Systems, Vol. 17, No. 4, pp. 162166, 1981.

[9] Y. Bar-Shalom, X. Li, and T. Kirubarajan, Estimation with Applications to Tracking and Navigation, Wiley, New York, 2001.

[10] L. Meiqin, Z. Di, and Z. Senlin, "Bearing-Only Target Tracking using Cubature Rauch-TungStriebel Smoother", In: Proc. of the 34th Chinese Control Conf., Hangzhou, China, pp.4734-4738, 2015.

[11] L.Badriasl and K. Dogancay, "ThreeDimensional Target Motion Analysis Using Azimuth/Elevation Angles", IEEE Transactions On Aerospace And Electronic Systems, Vol.50, No.4, pp. 3178-3194, 2014.

[12] D.V. Stallard, "Angle-only tracking filter in modified spherical coordinates", Journal of Guidance, Control, and Dynamics, Vol. 14, No.3, pp. 694-696, 1991.

[13] H.D. Hoelzer, Range Normalized Coordinates for Optimal Angle-Only Tracking in Three Dimensions, Teledyne-Brown Engineering, Huntsville, 1980.

[14] R.R. Allen and S.S. Blackman, "Angle-only Tracking With a MSC Filter", In: Proc. of Digital Avionics Systems Conf., IEEE, Los Angeles,USA, pp. 561-566, 1991.

[15] L. Qiang, S. Lihui, W. Hongxian, and G. Fucheng, "Utilization of Modified Spherical Coordinates for Satellite to Satellite BearingsOnly Tracking", Chinese Journal of space science, Vol. 29, No. 6, pp. 627-634, 2008.

[16] M. Mallick, L. Mihaylova, S. Arulampalam, and Y. Yan, "Angle-only Filtering in 3D Using Modified Spherical and Log Spherical Coordinates", In: Proc. of 14th International Conf. on Information Fusion, Chicago, USA, pp.1905-1912, 2011.

[17] B. Ristic, S. Arulampalam, and N. Gordon, "Beyond the Kalman Filter: Particle Filters for Tracking Applications", Artech House publishers, 2004.
[18] G. Ming-jiu, Y. Xiao, H. You, and S. Bao, "An Approach to Tracking a 3D-Target with 2DRadar", In: Proc. of IEEE International radar conf., Arlington, USA, pp. 763 - 768, 2005.

[19] T.R. Kronhamn, "Bearings-only target motion analysis based on a multi hypothesis Kalman filter and adaptive ownship motion control", In: IEE Proc. of Radar, Sonar Navigation, Vol. 145, No. 4, 1998.

[20] M. Mallick, Y. Bar-Shalom, T. Kirubarajan, and M. Morelande, "An Improved Single-Point Track Initiation Using GMTI Measurements", IEEE Transactions on Aerospace And Electronic Systems, Vol. 51, No. 4, pp. 26972713, 2015.

[21] B. Sindhu, J. Valarmathi, and S. Christopher, "Extended Kalman filter based range estimation using angle only measurements in 3D", In: Proc. of Global Conference on Advances in Science, Technology and Management, VIT University, Vellore.

[22] S. Julier, J. Uhlmann, and H.F. Durrant-Whyte, "A new method for the nonlinear transformation of means and covariances in filters and estimators", IEEE Transactions on Automatic Control, Vol. 45, No. 3, pp. 477-482, 2000.

[23] L. Zuo, R. Niu, and P.K. Varshney, "Conditional Posterior Cramér-Rao Lower Bounds for Nonlinear Sequential Bayesian Estimation", IEEE Transactions on signal processing, Vol. 59, No. 1, pp. 1-14, 2011.

[24] H. Dong and S. Chen, "Research of moving target tracking algorithm for video", International journal of intelligent Engineering and systems, Vol. 4, No. 1, pp. 18-25, 2011.

[25] N. Peach, "Bearings-only tracking using a set of range-parameterised extended Kalman filters", In: IEE Proc. of Control Theory and Applications, Vol. 142, No. 1, pp. 73-80, 1995.

[26] H. Wu, S. Chen, B. Yang, and X. Luo, "Rangeparameterised orthogonal simplex cubature Kalman filter for bearings-only measurements", IET Science, Measurement and Technology, Vol. 10, No. 4, pp. 370-374, 2016.

[27] E.A. Wan, and R. van der Merwe, "The Unscented Kalman Filter for Nonlinear Estimation", In: Proc. of IEEE Symposium (ASSPCC), Alberta, Canada, pp. 153-158, 2000.

[28] V.J. Aidala and S.E. Hammel, "Utilization of Modified Polar Coordinates for Bearings-Only Tracking", IEEE Transactions on automatic control, Vol. 28, No. 3, pp. 284-294, 1983.

[29] Y. Bar-Shalom, P. Willett, and X. Tian, Tracking and Data Fusion: A Handbook of Algorithms, YBS Publishing, New York, 2011. 
[30] T. Brehard and J. P. Le Cadre, "Closed-form Posterior Cramer-Rao Bound for a Maneuvering Target in the Bearings-Only Tracking Context Using Best-Fitting Gaussian Distribution", IEEE Transaction on Aerospace and Electronic Systems, Vol. 42, No. 4, pp. 1198-1223, 2006. 